\title{
ESTADO NUTRICIONAL Y PARASITOSIS POR ENTEROBIUS VERMICULARIS EN NIÑOS MENORES DE 5 AÑOS
}

\author{
NUTRITIONAL STATUS AND PARASITOSIS FOR ENTEROBIUS \\ VERMICULARIS IN CHILDREN UNDER 5 YEAR OLD \\ María del Rosario Farromeque Meza', Brunilda Edith León Manrique', \\ Carlos Roberto Pesantes Rojas ${ }^{1}$, Luis Alberto Aguirre Anaya ${ }^{1}$, Lily Yahaira Chávez Anaya ${ }^{1}$
}

\section{RESUMEN}

La investigación busco evaluar el estado nutricional y parasitosis por Enterobius vermicularis en niños menores de 5 años. En el método se tuvo la participación de 131 niños de 2 a 5 años ( 67 varones y 64 mujeres) del Centro Educativo Inicial Parroquial “Jesús Divino Maestro"- Manzanares, evaluándose medidas antropométricas (Talla, Peso), diagnostico parasitológico (método Graham), consumo de productos con mayor frecuencia en su alimentación y un diagnostico socio económico, encontrándose un 3,1\% con obesidad $6,1 \%$ con sobrepeso, $81 \%$ normal y $9,8 \%$ con desnutrición asimismo presentaron parasitosis por Enterobius vermicularis un $35 \%$, llegando a la conclusión que los niños con desnutrición presentaron un 13,7\% de parasitosis por Enterobirus vermicularis y los niños en estado normal $21,3 \%$.

Palabras clave: Estado nutricional; Enterobius vermicularis.

\begin{abstract}
The research sought to evaluate the nutritional status and parasitosis by Enterobius vermicularis in children under 5 years of age. The method involved the participation of 131 children from 2 to 5 years old ( 67 males and 64 females) of the "Jesús Divino Maestro" Parochial Initial Educational Center - Manzanares, evaluating anthropometric measurements (Size, Weight), parasitological diagnosis (Graham method), consumption of products with greater frequency in their diet and a socio-economic diagnosis, finding $3.1 \%$ with obesity $6.1 \%$ with overweight, $81 \%$ normal and $9.8 \%$ with malnutrition also presented parasitosis by Enterobius vermicularis $35 \%$, reaching the conclusion that children with malnutrition presented $13.7 \%$ of parasitosis by Enterobirus vermicularis and children in normal state $21.3 \%$.
\end{abstract}

Keywords: Nutritional status; Enterobius vermicularis.

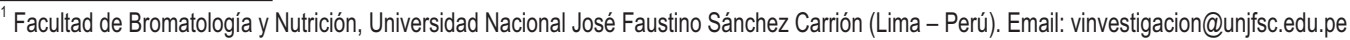




\section{INTRODUCCIÓN}

La desnutrición es una emergencia silenciosa e invisible y muy grave que cobra un precio muy alto en los niños, y sus familias es resultado de un gran número de causas entre las que podemos destacar la carencia de alimentos debido a la pobreza, las infecciones comunes en los niños y niñas en los primeros años de vida, las enfermedades diarreicas agudas (EDAs) e infecciones respiratorias agudas (IRAs), también tiene relación con las conductas o prácticas de salud de las madres, como su comportamiento con respecto a la lactancia exclusiva, ablactancia, higiene.(UNICEF 1998).

En cuanto, a las enfermedades parasitarias constituyen una de las causas de morbilidad más importantes en niños en toda Latinoamérica, que pone en riesgo la salud y vida de la población, tornándose en una carga económica tanto para la familia como para la sociedad. Las áreas más afectadas son las suburbanas y rurales tomando en cuenta el aspecto educativo. Las parasitosis intestinales son infecciones muy frecuentes (prevalencia del $40-70 \%$ ) en los países subdesarrollados constituye un problema de salud debido a que en ellos coexisten malas condiciones higiénicas, escasa educación sanitaria, deficiente saneamiento ambiental y bajas condiciones socio-economicas. En general tiene baja mortalidad, pero igualmente ocasionan importantes problemas sanitarios y sociales debido a su sintomatología y complicaciones (Botero 2005)

La prevalencia de Enterobius vermicularis en escolares de 05 a 12 años de edad de la comunidad de San Lorenzo-Datem del Marañon-Loreto; se evaluó en la Institución Educativa del nivel primaria, se utilizó dos métodos y fueron el método directo y el método de Graham, se hizo la comparación respectiva y se constató que el método ideal para esta parasitosis es el segundo método. La toma de muestra se llevó a cabo durante los meses de Junio y Julio 2010, analizándose un total de 120 muestras de escolares de la comunidad de San Lorenzo. Cabe mencionar que, por razones de ética en la investigación, se trabajo con los escolares que fueron autorizados por los padres que previamente firmaron el consentimiento informado. Se realizo una encuesta para determinar los síntomas más frecuentes de esta parasitosis y las prácticas higiénicas de los escolares de la comunidad de San Lorenzo. El muestreo se realizó en forma total, seriada (tres aplicaciones por escolar), durante tres días consecutivos. En el presente estudio se concluye que la prevalencia de Enterobius vermicularis alcanzó un índice de 15\%. Esta baja prevalencia encontrada de este parasito se debía a los factores climáticos de la región, a los buenos hábitos higiénicos de la población en estudio, a las condiciones sanitarias básicas existentes y al no hacimiento observado en los ambientes de la Institución Educativa Primaria de San Lorenzo.(Zevallos S.;F. 2010).

El parasitismo intestinal y malnutrición en niños residentes en una zona vulnerable de la ciudad de Santa María, Colombia, con el propósito de establecer la frecuencia de parasitismo intestinal y malnutrición en una población de 392 niños con edades entre 3 y 5 años, residentes en tres barrios subnormales de la ciudad de Santa María. Para evaluar la condición nutricional de los niños, se midieron los índices antropométricos a peso para la edad (RPE) y talla para la edad (RTE). La frecuencia de parásitos intestinales fue del $55.1 \%$ (216/392), los parásitos con potencial patogenicidad fueron Entamoeba histolytica (19.9\%), Giardia duodenalis $(12.7 \%)$, Blastocystis hominis $(11.7 \%)$, y Ascaris lumbricoides (10.7\%). El poli-parasitismo estuvo presente en el $17.3 \%$ de la población (65/392). La malnutrición aguda se observó en el $41.8 \%$ y la crónica en el $30.1 \%$ de los niños. Se encontró una asociación estadísticamente significativa entre parasitismo general y la RPE $(p=0.0235)$, pero la correlación entre el parasitismo y la RTE no fue significativa $(p=0.11)$. El presente resultado demuestra que la frecuencia de infecciones por parásitos intestinales es alta en la población infantil vulnerable de Santa María, no obstante, no es posible asegurar que exista una relación causaefecto entre el parasitismo y el déficit nutricional.

Por esta razón, se desarrolló la investigación Estado Nutricional y parasitosis por Enterobius vermicularis en niños del I.E.I..P. "Jesús Divino Maestro"- Manzanares que alberga niños menores de 5 años, procedente de sectores de pobreza y extrema pobreza donde factores como el bajo nivel cultural, hacinamiento, saneamiento básico deficiente, falta de higiene personal y colectiva, factores ambientales son un riesgo para la salud.

\section{METODOLOGIA}

Área de estudio: I.E.I.P.”Jesús Divino Maestro"

Tipo de Investigación: Descriptivo analítico

Nivel de Investigación: Básica

\section{MATERIALES Y METODOS}

Diagnostico Nutricional

Medidas antropométricas (Talla, Peso)

Ficha de Evaluación Nutricional

\section{Encuesta socio económica}

Diagnostico parasitológico (Método Graham)

Población: Se evaluó a una población de 131 niños de 2 a 5 años de edad del I.E.I.P. "Jesús Divino Maestro" Manzanares.

Muestra : 67 niños y 64 niñas.

\section{RESULTADOS}

La población en estudio estuvo conformada por 67 niños $(51.1 \%)$ y 64 niñas $(48.9 \%)$ 

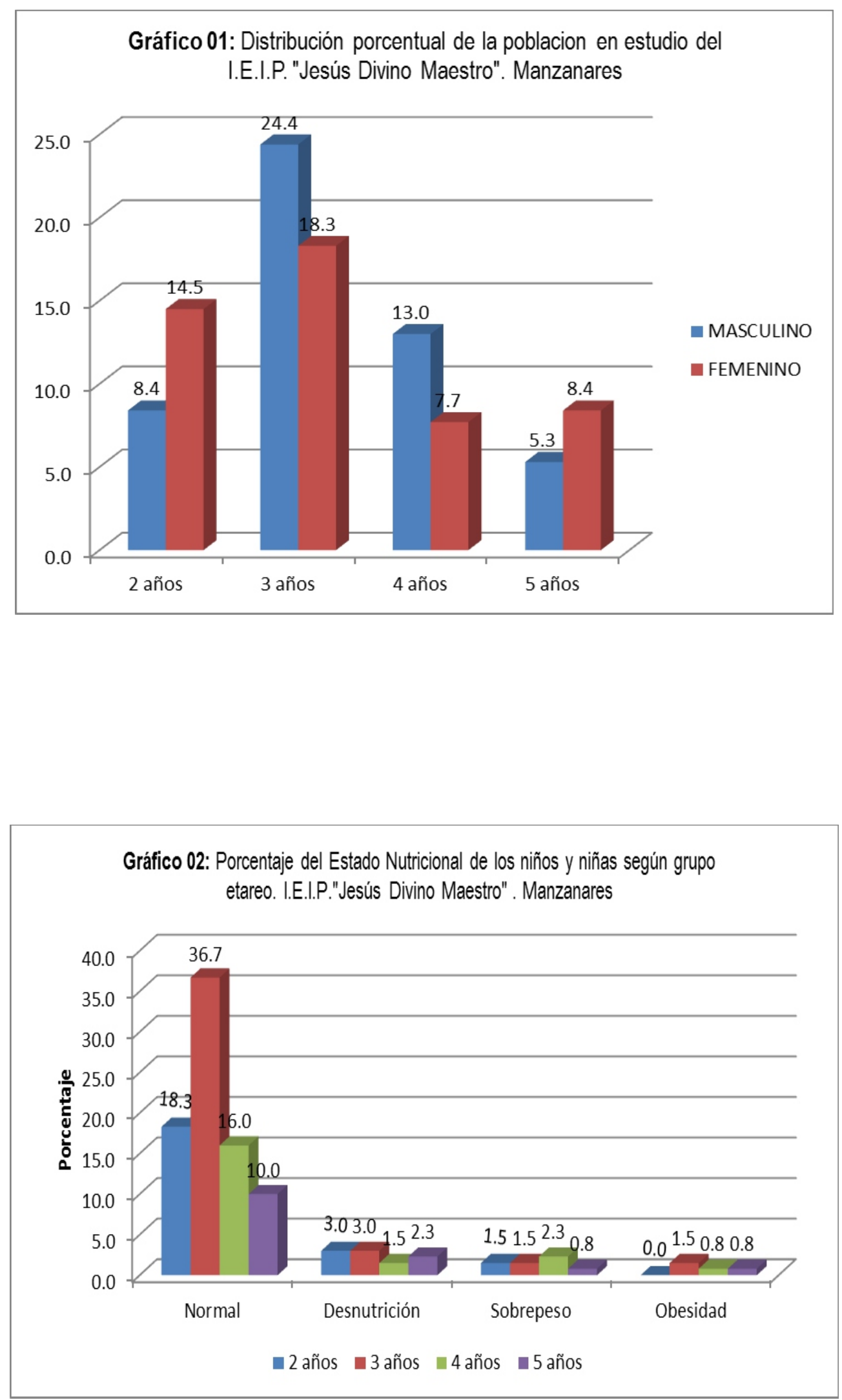

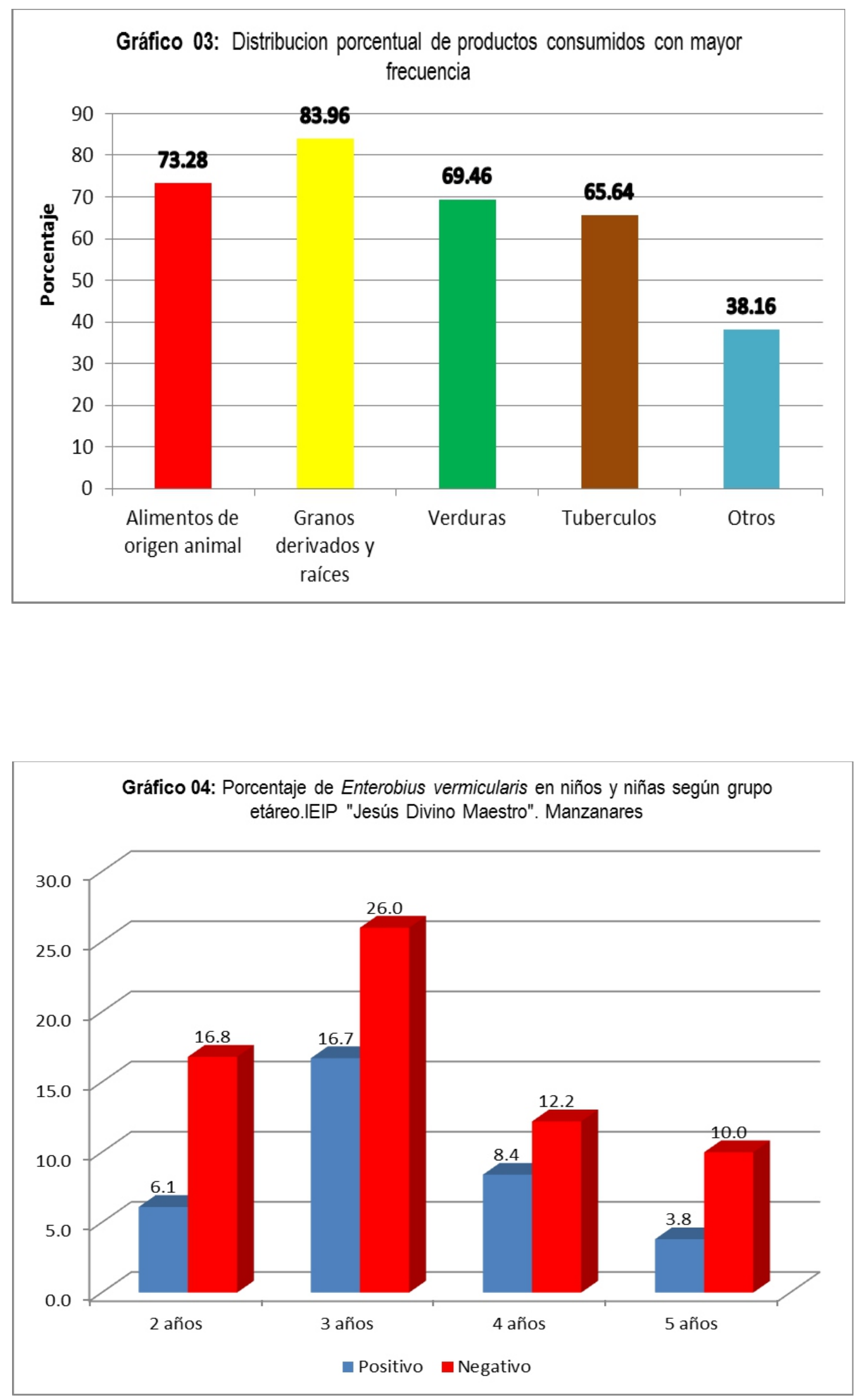

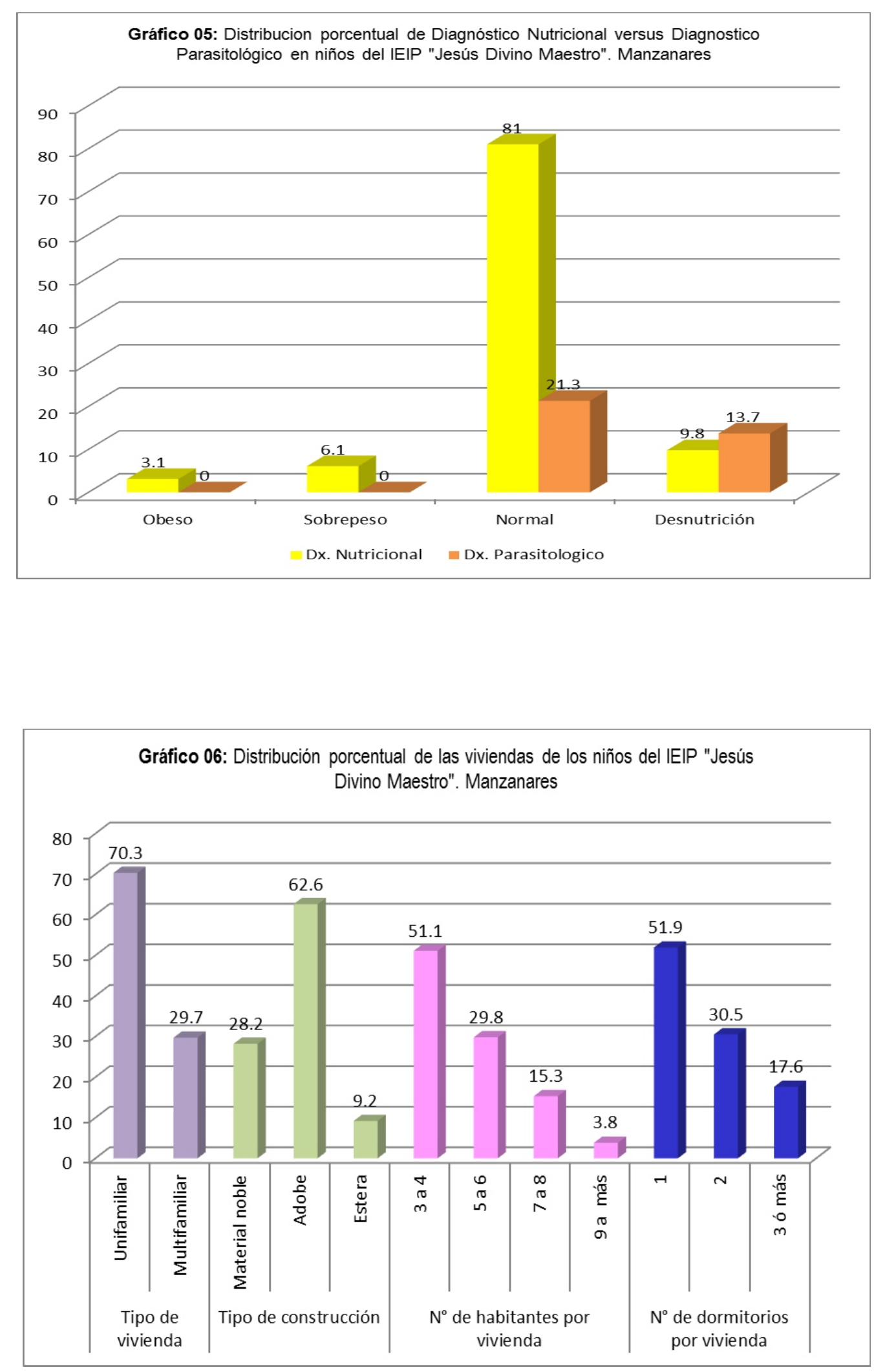


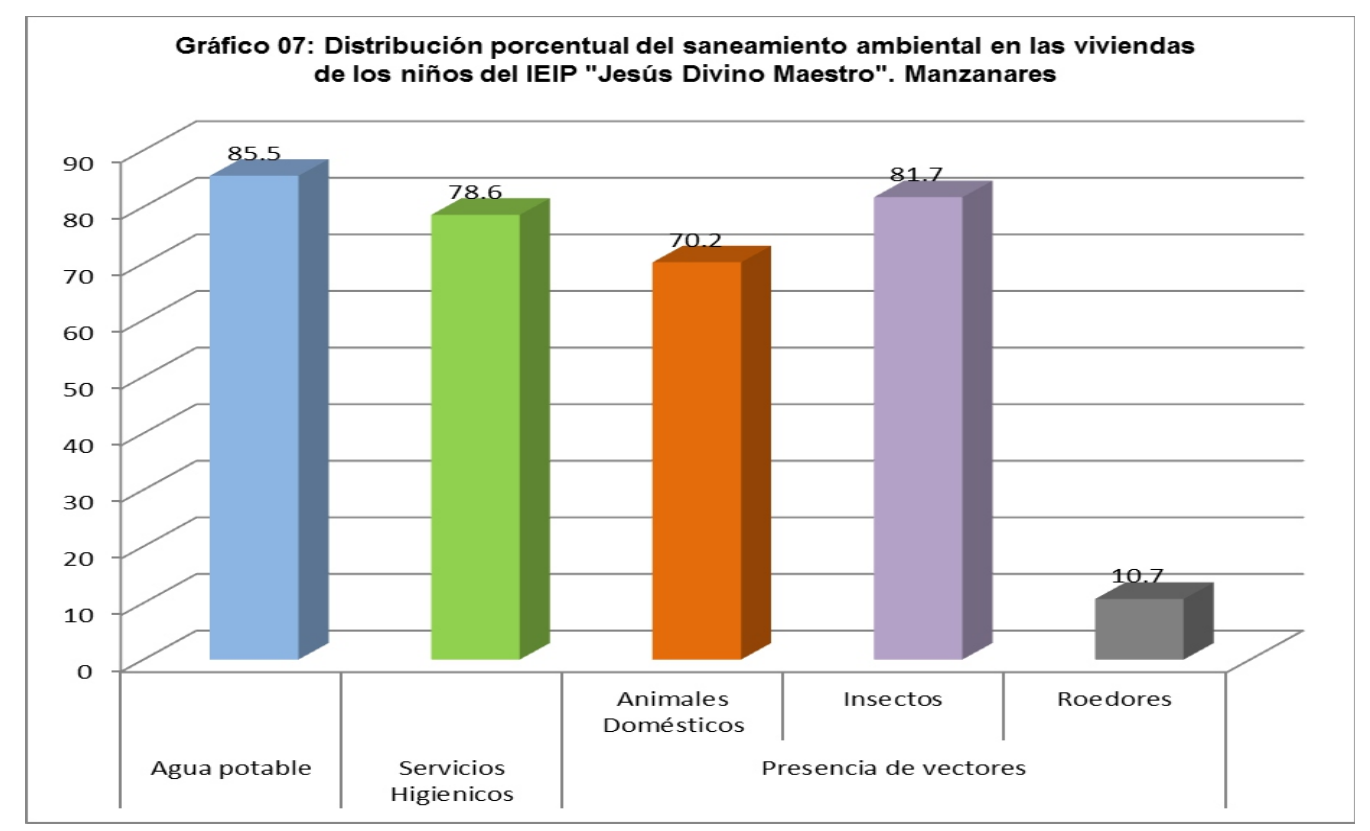

\section{DISCUSIÓN}

Los resultados obtenidos indican una parasitosis por Enterobius vermicularis en un $35 \%$, con ello las condiciones higiénicas de los niños, los juegos propios de la edad, el aseo personal influye en su salud, según lo manifestado por lannacone J. Benites M, Chirinos L. (2006) estas enfermedades de naturaleza infecciosaparasitaria pueden alterar el crecimiento y desarrollo de los niños. En cuanto al estado nutricional se tiene un $9,8 \%$ presentan desnutrición de los cuales el $13.7 \%$ se le diagnostico parasitosis por Enterobius vermicularis pero, cabe mencionar lo manifestado por Mendoza M.D: Lozano S.S (2010), que la frecuencia de infecciones por parásitos intestinales en la población infantil vulnerable no asegura que exista una relación causa efecto entre el parasitismo y el déficit nutricional. También es importante destacar lo mencionado por Rodriguez S. J; Contreras Q.M. (2015), que los factores sociales, habitos de higiene personal y saneamiento básico intradomiciliario con la incidencia de Enterobius vermicularis mostró una asociación significativa con un $p<0.05$, equivalente a una certeza mayor a $95 \%$.

\section{REFERENCIAS BIBLIOGRÁFICA}

UNICEF. 1998. Focus on Nutrition. Oxford Universitu Press.

Hammond K.A. 2001. Valoración Aliment.aria y clínica.
In Mahan LK, Escott-Stump S. Editores Nutricion y Dietoterapia de Kruse. Decima edición. Mexico. Mc Graw-Hill Interamericana.

Botero D. y Restrepo M. 2005. Parasitosis Humana.

Cuarta Edición. Medellín. Colombia. 85-87.

Lannacone J. Benites M, J. Chirinos L. 2006. Prevalencia de infección por parásitos intestinales en escolares de primaria de Santiago de Surco, Lima, Perú. Parasitologia Latinoam. 61 (1-2): 54-62.

Mendoza Meza Dary Luz y Lozano Socarras Sonia Liliana. 2010. Parasitismo intestinal y Malnutrición en niños residentes en una zona vulnerable de la ciudad de Santa Marta. Duazary. Diciembre. Vol.7 №2.

Rodriguez Soto Juan Carlos y Contreras Quiñones Marisol. 2015. Factores sociales e incidencia de Enterobius vermicuaris en instituciones educativas de nivel inicial del Distrito de Cascas. In Crescendo Institucional. 6(1): 11-21.

Martinez Rodrigo y Fernández Andrés. Desnutrición infantil en América Latina y el Caribe. Boletin de la infancia y adolescencia sobre el avance de los objetivos de desarrollo del Milenio. División de Desarrollo Social de la CEPAL. Número 2.

OMS. Tablas de valoración nutricional antropométrica. Organización Panamericana de la Salud. 